\title{
Maximum mistuning amplification of the forced response vibration of turbomachinery rotors in the presence of aerodynamic damping
}

\author{
Carlos Martelela,*, J. J. Sánchẹ-Álvarez ${ }^{\text {a }}$ \\ ${ }^{a}$ Depto. de Matemática Aplicada a la Ingenieria Aeroespacial, E.T.S.I. Aeronáutica y del \\ Espacio, Universidad Politécnica de Madrid, 28040 Madrid, Spain
}

\begin{abstract}
Mistuning can dangerously increase the vibration amplitude of the forced response of a turbomachinery rotor. In the case of damping coming from aerodynamic effects the situation is more complicated because the magnitude of the damping changes for the different travelling wave morles of the system. This damping variability modifies the effect of mistuning, and it can even result in a reduction of the mistunerl forced response amplitude below that of the tuned case (this is not possible in the usual case of constant material damping). In this paper the Asymptotic Mistuning Model (AMM) methodology is used to analyze this situation. The AMM is a reduced order model that is systematically derived from the mistuned bladed disk full model using a perturbative procedure based on the small size of the mistuning and the damping. The AMM allows to derive a very simple expression for an upper bound of the maximum amplification factor of the vibration amplitude that the system can experience (an extension of the well known Whitehead 1966 result to include the effect of non-uniform aerodamping). This new upper bound gives information on the mechanisms involved in the amplification/reduction of the mistuned response: (i) the number of modes participating in the response, and (ii) the ratio between the aerodamping of the directly forced mode and that of the of the rest of the modes. A FEM of a mistuned bladed disk is also used to verify the AMM predictions for several different forcing configurations, and both results show a very good quantitative agreement.
\end{abstract}

Keywords: Forced response, Mistuning, Aerodynamic damping, Turbomaxhinery 


\section{Introduction}

The term mistuning refers to the small differences among the (theoretically perfectly identical) blades in turbomachinery bladed disks. These small undesirable random imperfections result from manufacturing, assembling and material tolerances, as well as in-service wear; and are essentially unavoidable.

Despite of the fact that the magnitude of the mistuning is typically very small, it has a strong influence on the dynamical response of the bladed disk. There has been a lot of research since the 1970s on the effect of mistuning (details can be found in the review [1]), and, roughly speaking, it can be said that in the case of flutter it has a stabilizing effect, while for the forced response excitation it typically leads to a considerable amplification of the vibration amplitudes of individual blades. It has been reported in many previous experiments and simulations that the mistuned response can exceed the corresponding vibration amplitude of the tunerl structure (i.e., the structure without imperfections) by a factor that is frequently in the range from 1.5 to 2 (see, e.g., [2]). These increased blade vibration levels have a dramatic effect in terms of high cycle fatigue (HCF), which has been identified as the largest single cause of major component failures in aircraft gas turbine engines $[3,4,5]$. Mistuning therefore has a considerable negative impact on the safety, operability and readiness of aircraft gas turbine engines, and many research efforts have been directed to improve the understanding of this phenomenon and to try to predict and quantify its undesirable effects.

Fifty years ago Whitehead [6] introduced the following elegant and simple expression for the maximum amplification factor that the vibration amplitude of a forced mistuned bladed disk can experience

$$
\text { Amplification }_{\max }=\frac{1+\sqrt{N}}{2},
$$

with $N$ being the number of blades. The result above applies to a situation where the mode that is being forced belongs to a modal family with very similar modal frequencies (blade dominated), and the damping and the mistuning are small. This amplification factor is an upper bound and, according to the review of results presented in [2], it can be quite conservative for large values of $N$. But, despite of its accuracy, this kind of expressions are very useful from a preliminary analysis point of view because they give a quick idea of how bad things can get because of mistuning, and what are its basic dependencies and scalings.

More recently, Whitehead's result was updated using a reduced order model methodology called Asymptotic Mistuning Model (AMM) to obtain

$$
\text { Amplification } \max =\frac{1+\sqrt{N_{a}}}{2}
$$

where $N_{a}$ is now the number of active modes, that is, the number of modes that have freruencies close to the frequency of the forced mode (see $[7,8]$ ). The key 
idea from the AMM is that the important thing is not the total number of blades but the number of modes with frequencies similar to that of the forced mode; these are the only modes that the small mistuning can couple and combine to produce an increase of the forced response. This can be clearly seen for example in [9] where the number of blades is $N=64$ but the maximum mistuned amplification that is obtained is only about 1.2 because the number of active modes appears to be just $N_{a}=2$.

All the considerations above assume uniform material damping. If we now take into account aerodynamic effects, then the resulting aerodamping typically dominates over the material damping, being at least one order of magnitude larger [10]. This situation is particularly relevant for the case of BLISKS (integrally blarled rotors with no blade-disk interfaces), where there is no friction damping and nearly all the damping comes from the gas flow.

The effect of mistuning on the forced response of a rotor with aerodynamic damping is more complicated, and changes substantially with respect to the uniform material damping case. There is even the possibility of having a reduction (by a factor of 2 or more) of the forced response vibration ampliturle by the effect of mistuning, as it has been reported in $[11,12]$ and found in many configurations $[13,14,15,16]$. The reason for this change in the effect of mistuning relies in the fact that the aerodynamic damping has different values for the different travelling wave (TW) modes of the tuned structure. If the TW that is being forced has small aerodynamic damping, then mistuning will couple it with other TWs with higher damping and the mistuned response will have a higher effective damping, which will translate directly into a reduction of the vibration amplitude.

The analyses available in the literature of the forced response of a mistuned rotor with aerodynamic damping typically perform statistical explorations and optimizations on full FEM models and reduced order models $[11,12,14,16]$. The purpose of this paper is to analyze the effect of mistuning using the AMM methodology $[17,7,8]$. The resulting AMM is a very reduced model that gives information about the basic mechanisms that play a role in the mistuned response. One interesting outcome of the AMM analysis is the following expression for the maximum response amplification

$$
\text { Amplification }_{\max }=\frac{1+\sqrt{\xi_{r} \sum_{a} \frac{1}{\xi_{u}}}}{2},
$$

where $\xi_{r}$ is the aerorlamping of the TW that is being directly forced, $\xi_{a}$ is the aerodamping of an active TW modes, and the sum goes over all $N_{a}$ active modes. This upper bound can be regarded as an extension of Whitehead's but now including the variability of the aerodamping from TW to TW (if the aerodamping is set to constant then Whitehead's result is recovered). It is very simple, but it gives information on the two mechanisms that have an effect on the amplification of the mistuned response: (i) the number of active TW morles involved in the response (as in Whitehead's expression), and (ii) the ratio between the aerodamping of the forced morle, $\xi_{r}$, and that of the of the 
rest of the active modes (this was not present in the case of uniform damping). This expression with $N_{a}$ set to $N$ (number of blades) resembles also the one obtained by Chan \& Ewins [18] for the maximum blade vibration of a singleDOF-per-sector system with variable blade structural damping; but there is an important difference: in their case what appears in the expression for the maximum response is the damping coefficient of the blades, while here it is the TW damping.

The remaining of this paper is organized as follows. The AMM for the mistuned forced response with aerodynamic damping is first derived in the next section. The AMM is then used to pose and solve an optimization problem that gives the maximum response amplification. This is followed by a verification of the AMM results against those from a FEM morlel of a simplified blisk for two characteristic forcing configurations. And, finally, some conclusions are drawn in the last section.

\section{AMM of the Forced Response problem}

In order to keep this paper self-contained the derivation of the AMM is briefly explained in the Appendix, here we just present the resulting reduced model equations (more interested readers can find the details in $[17,7,8]$ ).

The basic irlea behind the AMM is that, for TW forcing and near resonance, the only modes that can be couplerl through mistuning and participate in the forcerl response of the system are those with frequency close to that of the morle that is being directly forced (that is, close to the tuned resonant frequency). These modes are called "active modes", and the remaining modes, which do not have a substantial effect in the response, are referred to as "passive modes".

The resulting AMM is an extremely simplified description of the system mistuned response near the tuned resonance frequency (it does not cover larger frequency sweeps as other ROMs do $[19,20]$ ), and it can be written as a system of linear equations

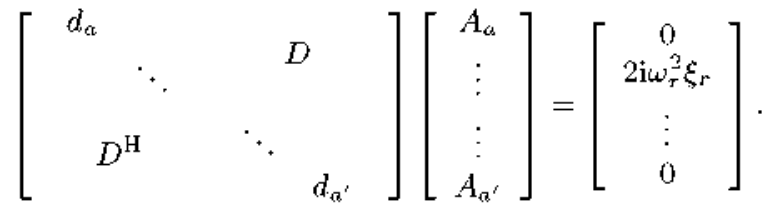

for the amplitudes of the $N_{a}$ active morles $A_{a}, \ldots, A_{a^{\prime}}$. The diagonal terms are given by

$$
d_{a}=2 \omega_{r}^{2}\left(\Delta \omega_{a}-\Delta \omega\right)+2 \omega_{a}^{2}\left(\eta_{a}+i \xi_{a}\right),
$$

where $\omega_{r}$ is the frequency of the TW mode directly forced (with engine order $r), \omega=\omega_{r}(1+\Delta \omega)$ is the forcing frequency, $\omega_{a}=\omega_{r}\left(1+\Delta \omega_{a}\right)$ is the frequency of the active modes, and $\eta_{a}$ and $\xi_{a}$ is the aerodynamic frequency correction and

damping of all active modes. The off-diagonal elements in $D$ are associated to mistuning and take the form:

$$
D_{a a^{\prime}}=Z_{a}^{\mathrm{H}}\left(\Delta K_{a-a^{\prime}}^{\mathrm{F}}-\omega_{r}^{2} \Delta M_{a-a^{\prime}}^{\mathrm{F}}\right) Z_{a^{\prime}},
$$


where $Z_{a}$ and $Z_{a^{\prime}}$ are the in-sector mode shape of axtive modes $a$ and $a^{\prime}$, and $\Delta K_{a-a^{\prime}}^{\mathrm{F}}, \Delta M_{a-a^{\prime}}^{\mathrm{F}}$ are the $\left(a-a^{\prime}\right)$ Fourier coefficients of the sector to sector mass and stiffness mistuning distribution. The external forcing has been also rescaled to make the maximum tuned response equal to one.

\section{Maximum amplification of the forced response}

The AMM formalism of the previous section is used in this section to derive an closed expression for the maximum mistuning amplification factor in the case of aerorlynamic damping.

The displacement of a generic point $p$ on sector $j$ can be expressed as

$$
\left|X_{j p}\right| \propto\left|\sum_{a=1}^{N_{\alpha}} A_{a} Z_{a} \mathrm{e}^{\mathrm{i}\left(\frac{2 \pi_{a}}{N}\right) j}\right|
$$

where the sum goes over all $N_{a}$ active morles, $A_{a}$ is the ampliturle of active morle $a$, and $Z_{a}$ is the corresponding in-sector mode shape at $p$ (see erl. (A.10)). If all active modes have very similar in-sector mode shapes, that is, if the modal family that is being forced is a blade dominated family whose vibration modes approximately differ only on their inter blade phase angles, then the displacement can be simplified to

$$
\left|X_{N p}\right| \propto\left|\sum_{a=1}^{N_{a}} A_{a}\right|
$$

where the sector has been set to $j=N$ to remove also the inter blade phase angle term.

The energy balance equation from the AMM is obtained pre-multiplying system (1) by the transpose conjugate of the vector of amplitudes

$$
\left[\bar{A}_{a}, \ldots, \bar{A}_{a^{\prime}}\right]
$$

and keeping only the imaginary part, to obtain

$$
\sum_{a=1}^{N_{a}} \omega_{a}^{2} \xi_{a}\left|A_{a}\right|^{2}=\omega_{r}^{2} \xi_{r} \operatorname{Re}\left(A_{r}\right)
$$

which basically says that the energy pumped into the system by the forcing has to be dissipated by the aerodynamic damping of the active modes (here $\operatorname{Re}()$ stands for the real part).

The optimization problem is then to maximize the displacement given by expression (4) subject to the energy constrain (5). In order to more easily work out its solution it is convenient to split the complex amplitudes into real and imaginary part

$$
A_{a}=x_{a}+\mathrm{i} y_{a}
$$


to rewrite the problem with real variables as

$$
\begin{array}{rc}
\text { maximize } & \left(\sum_{a=1}^{N_{a}} x_{a}\right)^{2}+\left(\sum_{a=1}^{N_{a}} y_{a}\right)^{2} \\
\text { with the constrain } & \sum_{a=1}^{N_{a}} \omega_{a}^{2} \xi_{a}\left(x_{a}^{2}+y_{a}^{2}\right)=\omega_{r}^{2} \xi_{r} x_{r}
\end{array}
$$

The corresponding Lagrange function takes the form

$$
L=\left(\sum_{a=1}^{N_{a}} x_{a}\right)^{2}+\left(\sum_{a=1}^{N_{a}} y_{a}\right)^{2}-\lambda\left(\sum_{a=1}^{N_{a}} \omega_{a}^{2} \xi_{a}\left(x_{a}^{2}+y_{a}^{2}\right)-\omega_{r}^{2} \xi_{r} x_{r}\right)
$$

where $\lambda$ is the Lagrange multiplier associated with the energy constrain. Setting the grarlient of $L$ to zero gives the following $2 N_{a}$ equations for the optimal point

$$
\begin{array}{cl}
x_{a}=S_{x} /\left(\lambda \omega_{a}^{2} \xi_{a}\right) & \text { for } a=1, \ldots, N_{a}, \quad a \neq r \\
x_{r}=S_{x} /\left(\lambda \omega_{r}^{2} \xi_{r}\right)+\frac{1}{2} & \\
y_{a}=S_{y} /\left(\lambda \omega_{a}^{2} \xi_{a}\right) & \text { for } a=1, \ldots, N_{a}
\end{array}
$$

where $S_{x}=\sum_{a=1}^{N_{x}} x_{a}$, and $S_{y}=\sum_{a=1}^{N_{a}} y_{a}$. The values of $S_{x}$ and $S_{y}^{\prime}$ in terms of $\lambda$ can be obtained summing up all the above equations for $x_{a}$ and $y_{a}$

$$
\begin{aligned}
& S_{x}\left(\lambda-\sum_{a=1}^{N_{a}} \frac{1}{\omega_{a}^{2} \xi_{a}}\right)=\frac{\lambda}{2} \\
& S_{y}\left(\lambda-\sum_{a=1}^{N_{a}} \frac{1}{\omega_{a}^{2} \xi_{a}}\right)=0
\end{aligned}
$$

The last equation implies that $S_{y}=0$ and, according to eq. (10),

$$
y_{1}=0, \ldots, y_{N_{a}}=0
$$

which indicates that all TW modes are in phase at the maximum. The energy constrain (7), simplifies then to

$$
S_{x}^{2} \sum_{a=1}^{N_{a}} \frac{1}{\omega_{a}^{2} \xi_{a}}=\frac{1}{4} \omega_{r}^{2} \xi_{r} \lambda^{2}
$$

where we have also made use of eqs. (8) and (9) to eliminate all $x_{a}$. This last equation together with eq. (11) allows to compute the value of $S_{x}$, which is precisely the maximum displacement (see eq. (6)), and is given by

$$
\text { Amplification }_{\max }=\frac{1+\sqrt{\omega_{r}^{2} \xi_{r} \sum_{a} \frac{1}{\omega_{a}^{2} \xi_{a r}}}}{2}
$$

(recall that in the tunerl case only the amplitude $A_{r}$ is present, and its maximum displacement is $\left|A_{r}\right|=1$ ).

Finally, if we assume that all tuned TW mode frequencies are very similar, the expression above can be reduced to the slightly simpler form used in the Introduction:

$$
\text { Amplification }_{\max }=\frac{1+\sqrt{\xi_{r} \sum_{a} \frac{1}{\xi_{u}}}}{2} .
$$




\section{Mistuned BLISK validation}

The results from the AMM are validated in this section using a blisk model. It is a simplified blisk with $N=21$ sectors that is represented in Fig. 1 together with the FEM mesh of one of its sectors. The blisk FEM has approximately 3600 DOFs/sector, and the forced response of the complete blisk ( $\sim 7510^{3}$ DOFs) can be computed in a reasonable time.

The modal vibration frequencies of the tuned blisk are plotted in Fig. 2, which shows also the two very different forcing conditions that will be considered: 2nd family $\mathrm{EO}= \pm 2$, and 1st family $\mathrm{EO}= \pm 8(\mathrm{EO}=$ engine order $)$.

(a)

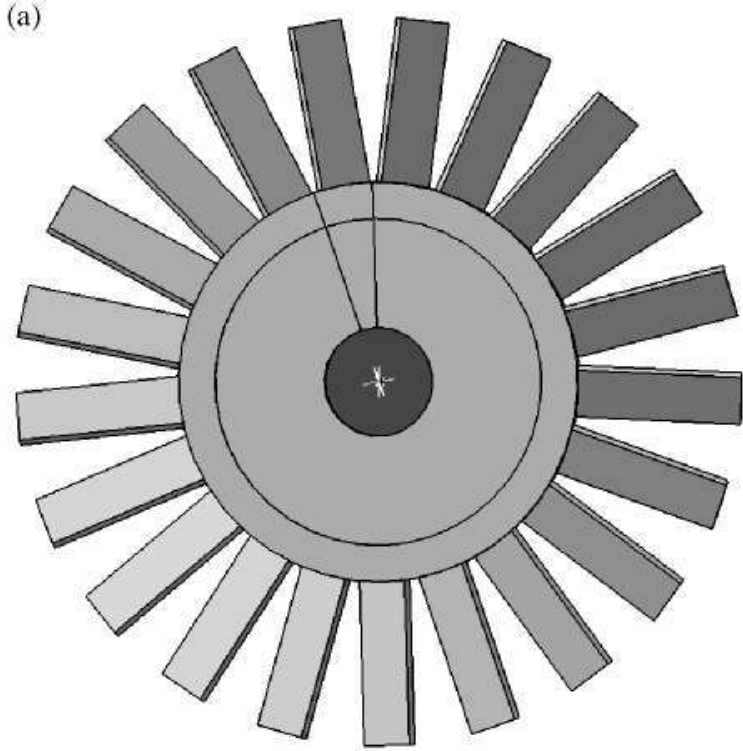

(b)

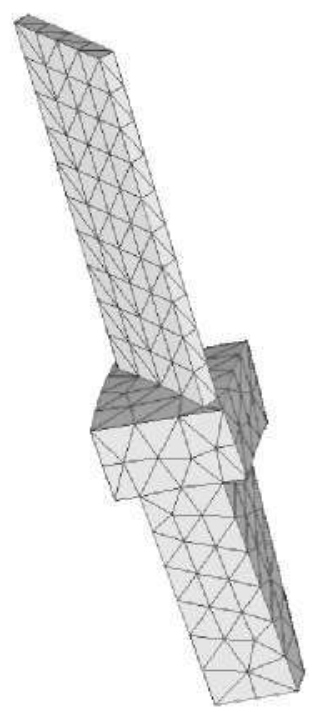

Figure 1: (a) Blisk model. (b) Sector mesh.

The matrix of the aerodynamic forces is a cyclic matrix designed to produce exactly the same aerodynamic correction to all family modes

$$
\mathbf{M}_{\text {aero }}=-2 c_{a}\left[\begin{array}{ccccc}
c_{0} K+K_{\mathrm{c}} & c_{0} K_{\mathrm{c}} & \ldots & K_{\mathrm{c}}^{T} & c_{0} K_{\mathrm{c}}^{T}+K \\
\ddots & \ddots & \ddots & \ddots & \\
K_{\mathrm{c}}^{T} & c_{0} K_{\mathrm{c}}^{T}+K & c_{0} K+K_{\mathrm{c}} & c_{0} K_{\mathrm{c}} & \\
& \ddots & \ddots & \ddots & \\
c_{0} K_{\mathrm{c}} & & K_{\mathrm{c}}^{T} & c_{0} K_{\mathrm{c}}^{T}+K & c_{0} K+K_{\mathrm{c}}
\end{array}\right] \text {, }
$$

where $c_{0}=\frac{1}{2}+\frac{3}{2} \mathrm{i}$, and the coefficient $c_{a}=0.001$ measures the magnitude of the aerodynamic effects. The resulting small frequency correction and aerodynamic damping for a modal family are shown in Fig. 3. Note that the blisk is stable (no flutter present) because all damping values are positive, and the TW mode 


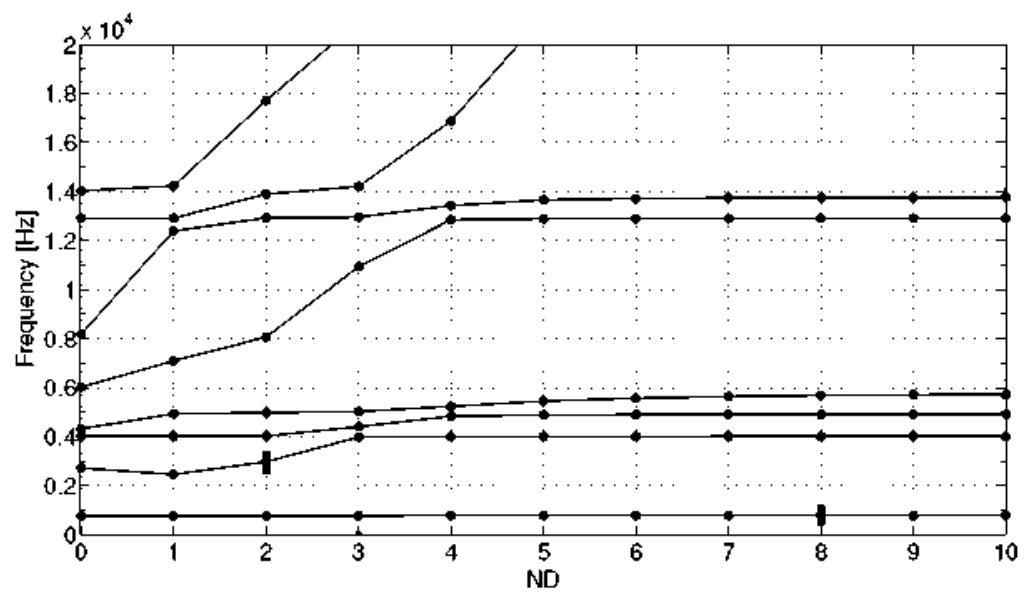

Figure 2: First eight families of the natural vibration frequencies of the tuned blisk. Thick lines indicate the forcing cases considered with engine order $\mathrm{EO}= \pm 2$ and $\mathrm{EO}= \pm 8$.

with lowest damping is that with nodal diameter $N D=5$. The distributions of aerodynamic frequency correction and damping used in this paper are given by a very simple toy model (constant value plus the first ND harmonic, see Fig. 3), which has been introduced with the idea of capturing just some of the basic characteristics of the data shown in Figure 4 of [16]: higher damping for negative TW index, lower for positive TW index, and a damping level below 0.0025 .

(a)

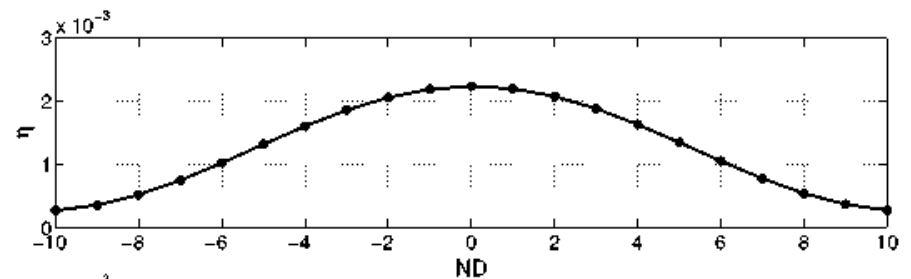

(b)

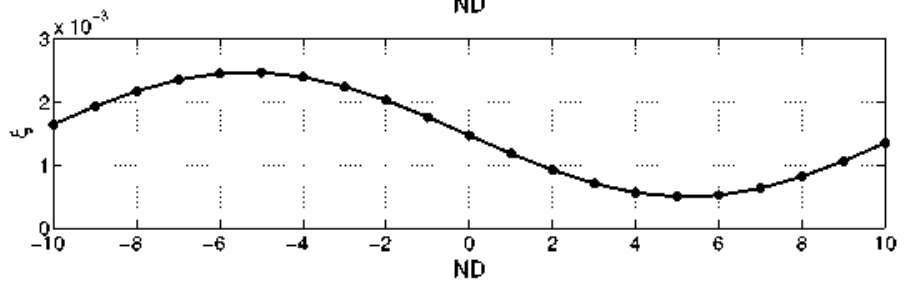

Figure 3: Aerodynamic frequency correction $\eta$ (a) and damping $\xi$ (b).

The mistuning effects are modeled including a small perturbation proportional to the blade stiffness of each sector. The mistuning matrices are of the 
form

$$
\Delta \mathbf{M}=0, \quad \Delta \mathbf{K}=\left[\begin{array}{cccc}
m(1) K_{m i s t} & 0 & \cdots & 0 \\
0 & m(2) K_{m i s t} & & \vdots \\
\vdots & & \ddots & 0 \\
0 & \cdots & 0 & m(N) K_{m i s t}
\end{array}\right]
$$

where $K_{\text {mist }}$ is the sector stiffness mistuning matrix that only includes the blade stiffness

$$
K_{\text {mist }}=\left[\begin{array}{cc}
K_{b b} & 0 \\
0 & 0
\end{array}\right],
$$

and the small numbers $m(1), \ldots, m(N)$ represent the sector-to-sector mistuning distribution around the blisk.

And finally, the forcing applied to the blisk is modeled as a TW with engine order EO and frequency $\omega$

$$
\mathbf{F}=\left[\begin{array}{c}
F \mathrm{e}^{\mathrm{i}\left(\frac{2 \pi}{N} \mathrm{E} O\right) 1} \\
F \mathrm{e}^{\mathrm{i}\left(\frac{2 \pi}{N} \mathrm{E} O\right) 2} \\
\vdots \\
F \mathrm{e}^{\mathrm{i}\left(\frac{2 \pi}{N} \mathrm{EO}\right) N}
\end{array}\right] \mathrm{e}^{\mathrm{i} \omega t}+c . c .
$$

with the following in-sector structure

$$
F=\left[\begin{array}{c}
X_{53} \\
0
\end{array}\right]
$$

which corresponds to a forcing on the blade DOFs equal to the displacements of mode 3 of the 5 th family, and no forcing on the disk.

Foreing with $E O=2$

The first case considered corresponds to forcing with $\mathrm{EO}=2$ near the resonance frequency of the second family, see Fig. 2. If we look close to the tuned resonant frequency, it is clearly seen that this mode is well separated from the rest. The only possible active modes are the TW with 2 nodal diameters (the one that is being directly excited by the forcing) and its symmetric TW corresponding to -2 nodal diameters, which has exactly the same frequency.

For this isolated mode forcing case, the AMM can be written in its simplest possible form (see $[17,7,8]$ ), with only two active TWs,

$$
\left[\begin{array}{cc}
-\Delta \omega+\left(\eta_{+}+\mathrm{i} \xi_{+}\right) & D \\
\bar{D} & -\Delta \omega+\left(\eta_{-}+\mathrm{i} \xi_{-}\right)
\end{array}\right]\left[\begin{array}{c}
A_{+} \\
A_{-}
\end{array}\right]=\left[\begin{array}{c}
\mathrm{i} \xi_{+} \\
0
\end{array}\right]
$$

where $\Delta \omega=\left(\omega-\omega_{0}\right) / \omega_{0}, \omega_{0}$ is the resonant tuned frequency corresponding to the TW of the second modal family with 2 nodal diameters (see Fig. 2), the 
sub-indexes + and - stand for the TW with $N D=2$ and $N D=-2$, and all the effect of mistuning is contained in the off-diagonal coefficient (see eq. (A.28))

$$
D=Z_{+}^{\mathrm{H}}\left(\Delta K_{4}^{\mathrm{F}}-\omega_{10}^{2} \Delta M_{4}^{\mathrm{F}}\right) Z_{-} /\left(2 \omega_{0}^{2}\right) .
$$

Note that only the harmonic with wavenumber 4 of the sector-to-sector mistuning distribution appears in the AMM formulation; the rest of the harmonics of the mistuning pattern have no effect on the response of the system.

The mistuning coefficient, $D$ in eq. (15) can be better understood if we take a look at the change in the modal vibration frequencies produced by mistuning when no forcing and no aerodynamic effects are included. These frequencies can be easily computer just by setting $\xi_{ \pm}=0$ and $\eta_{ \pm}=0$ (no forcing and no aerodynamic effects) in the above system (14) to obtain

$$
\left[\begin{array}{cc}
-\Delta \omega & D \\
\bar{D} & -\Delta \omega
\end{array}\right]\left[\begin{array}{l}
A_{+} \\
A_{-}
\end{array}\right]=\left[\begin{array}{l}
0 \\
0
\end{array}\right]
$$

This is now an eigenvalue problem for $\Delta \omega$ that gives the correction of the elastic modal frequencies due to mistuning: $\Delta \omega= \pm|D|$. According to eq. (A.22), this corresponds to two mistuned modal frequencies that can be approximately written as: $\omega_{+}=\omega_{0}(1+|D|)$ and $\omega_{-}=\omega_{0}(1-|D|)$. The magnitude of the mistuning coefficient $D$ can be therefore regarded as a measure of the relative frequency splitting produced by mistuning.

The AMM model above is extremely simple. The amplification of the response that is produced depends only on the size of the mistuning coefficient $|D|$ (see ec. (15)), and it is represented in Fig. 4. As the mistuning is increased, the amplification first shows a slight growth and then decays to a value near 0.6. This strong reduction of the maximum amplification of the forced response due to mistuning is a very interesting result that was recently reported in [11, 12].

The mechanism behind this reduction of the vibration amplitude can be easily explained by just looking at the AMM equations (14). The coupling due to the mistuning of the $N D=2$ TW (which has smaller damping, see Fig. 3) with the $N D=-2 \mathrm{TW}$ (with larger damping) results in an increase of the overall damping and, therefore, the response is reduced. This effect competes with the capability of the mistuning for increasing the response just by combining the two morles (as it happens in the uniform damping case, see [7]) and produces the initial small maximum of the curve. The circumstances under which there is amplification or reduction of the forced response were revealed and explained in $[11,12]$.

The maximum amplification (obtained using eq. (13)) takes the following simplified form for the case of just two active modes

$$
\text { Amplification }_{\max }=\frac{1+\sqrt{\xi_{+}\left(\frac{1}{\xi_{+}}+\frac{1}{\xi_{-}}\right)}}{2} .
$$

The dependence of the maximum amplification only on the ratio of the dampings $\xi_{+} / \xi_{-}$was alrearly detecterl in [21] where the mistuning effects on a two mode system were explored numerically. 


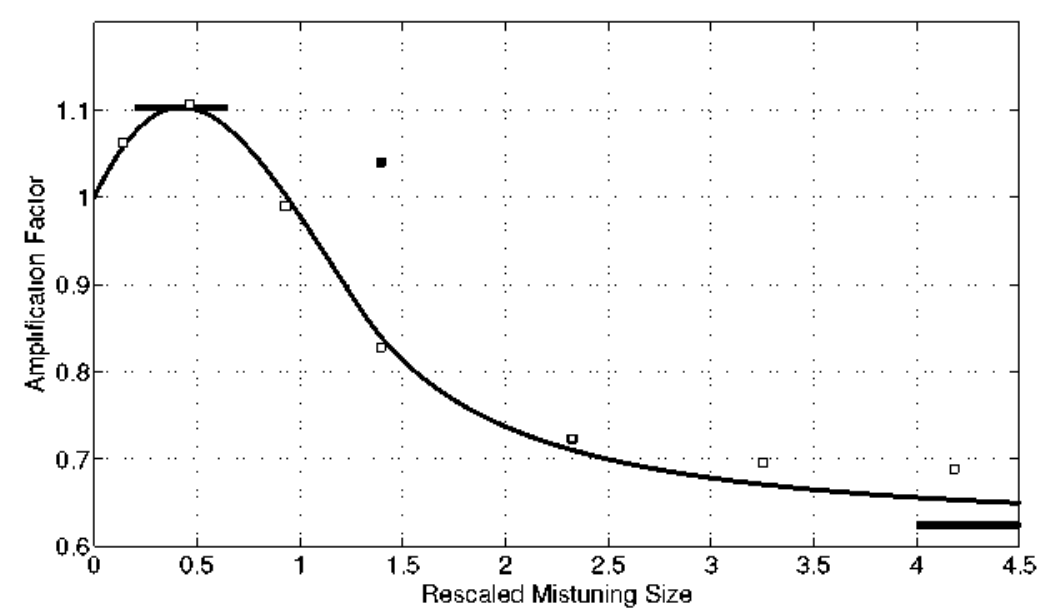

Figure 4: $\mathrm{EO}=2$ forcing. Amplification factor vs rescaled mistuning size $|D| / c_{\alpha}$ from the AMM. Thick lines correspond to the maximum amplification value eq. (16) and to the large mistuning value eq. (17). The squares mark solutions of the full FEM of the mistuned blisk.

The AMM formulation (14) is so simple in this case that it can be solved explicitly, and the resulting amplification factor can be written as

$$
\begin{aligned}
\mathrm{AF} & =\left|A_{+}+A_{-}\right|= \\
& =\frac{\left|\bar{D}-\left(\Delta \omega+\left(\eta_{-}+\mathrm{i} \xi_{-}\right)\right)\right| \xi_{+}}{\left.|| D\right|^{2}-\Delta \omega^{2}+\Delta \omega\left(\eta_{+}+\eta_{-}+\mathrm{i}\left(\xi_{+}+\xi_{-}\right)\right)-\left(\eta_{+}+\mathrm{i} \xi_{+}\right)\left(\eta_{-}+\mathrm{i} \xi_{-}\right) \mid}
\end{aligned}
$$

The above expression can now be used to explore the limit of large mistuning. To be more precise, if the modulus and phase of the mistuning coefficient are introduced $D=|D| \mathrm{e}^{\mathrm{i} \phi}$, the limit considered corresponds to $|D| \rightarrow \infty$ with $\eta_{ \pm}, \xi_{ \pm}$fixed and $\phi, \Delta \omega$ appropriately selected to maximize the result. The limit value of $\mathrm{AF}$ can only be different from 0 if $\Delta \omega$ verifies $\Delta \omega= \pm|D|+\Delta \omega^{\prime}$, with $\left|\Delta \omega^{\prime}\right| \ll|D|$ (which means that the forcing frequency has to be near one of the mistuning split frequencies), and then it reduces to

$$
\lim _{|D| \rightarrow \infty} \mathrm{AF}=\frac{\left|1 \pm \mathrm{e}^{\mathrm{i} \phi}\right| \xi_{+}}{\left|-2 \Delta \omega^{\prime}+\left(\eta_{+}+\eta_{-}+\mathrm{i}\left(\xi_{+}+\xi_{-}\right)\right)\right|},
$$

which is maximized for $\Delta \omega^{\prime}=\left(\eta_{+}+\eta_{-}\right) / 2$ and for $\phi=0, \pi$ to give

$$
\mathbf{A F}=\frac{2 \xi_{+}}{\xi_{+}+\xi_{-}} .
$$

The value of the maximum amplification factor from (16) and the large mistuning value from (17) are plotted as thick lines in Fig. 4.

The response of the full FEM problem has been computerl for the mistuning patterns

$$
m(j)=\varepsilon \operatorname{randl}(j)
$$




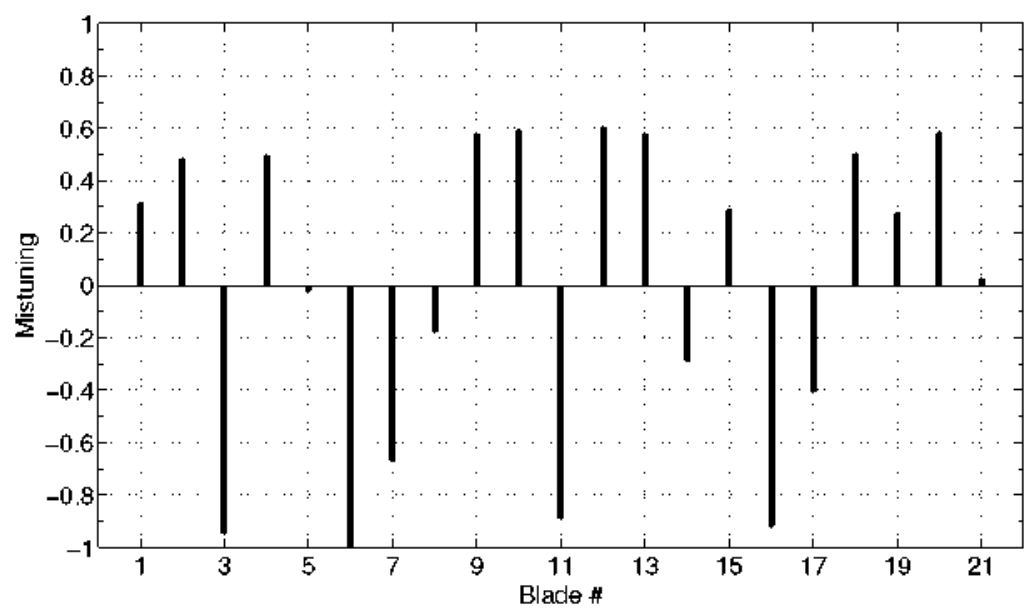

Figure 5: Random mistuning pattern rand( $j)$.

where $\operatorname{rand}(j)$ is a uniform random distribution with values in the range $[-1,1]$ plotted in Fig. 5 , and with the 7 mistuning amplitudes (relative to the magnitude of the aerodynamic effects)

$$
\varepsilon / c_{a}=0.03,0.1,0.2,0.3,0.5,0.7,0.9 .
$$

The results are represented in Fig. 4 as white square dots, and show a very good agreement with the AMM results. For larger values of the mistuning the FEM solution deviates from the AMM because it starts to feel the effect of other modal families that are not included in the AMM description. Another full FEM computation has been performed for the same random mistuning pattern with $\varepsilon / c_{a}=0.3$ but with the harmonic 4 removed. In this case, the resulting amplification shows no reduction and approaches the tuned value 1 (see black dot in Fig. 4), indicating that, as predicted by AMM methodology, this mistuning pattern has a much smaller effect in the forced response of the system.

\section{Forcing with $\mathrm{EO}=-\mathrm{2}$}

The situation is completely similar to the previous case, but the forcing is now acting on the $N D=-2 \mathrm{TW}$, which has higher aerodynamic damping (see Fig. 3). The resulting AMM formulation is exactly the same as in (14) but with the forcing appearing in the equation for $A_{-}$with the value $\mathrm{i} \xi_{-}$.

The amplification factor computed using the AMM is shown in Fig. 6. The behavior is now completely different from the previous case: as the mistuning level is increaserl there is first a strong growth of the maximum amplification, and then it becomes stabilized at a value slightly below 1.4. The amplification is now much higher than in the case of constant damping, which is limiterl to 
approximately $1.2[7]$. The strong amplification is again caused by the mistuning induced coupling that, in this case, results in a reduction of the effective damping that boosts the amplitude of the forced response.

The expressions for the global maximum amplification and for the maximum amplification for large mistuning can be obtained from (16) and (17) just by interchanging the sub indexes + and - . The resulting values happen to be very similar for this particular configuration and are plotted with thick lines in Fig. 6 .

The numerical results from the full FEM model with the random mistuning pattern given by (18) are again in good agreement with AMM results (white dots in Fig. 6); with a deviation that grows for larger mistuning values. And again, the random mistuning pattern with zero content in the harmonic 4 shows a response much closer to the tuned case (black dot in Fig. 6).

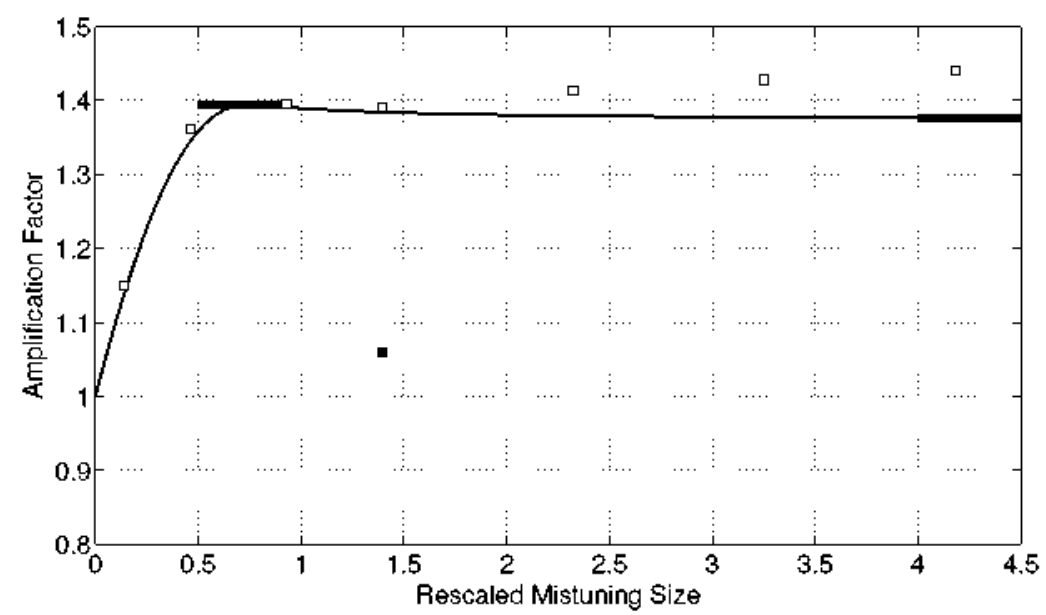

Figure 6: $\mathrm{EO}=-2$ forcing. Amplification factor vs rescaled mistuning size $|D| / c_{a}$ from the AMM. Thick lines correspond to the maximum amplification value (16) and to the large mistuning value (17). The squares mark solutions of the full FEM of the mistuned blisk.

Foreing with $E O=8$ and $E O=-8$

Two more forcing cases are consirlered with $\mathrm{EO}=8$ and $\mathrm{EO}=-8$, and with a frequency close to that of the first modal family (see Fig. 2).

The AMM includes now $N_{a}=21$ active modes; all modes in the first modal family have frequencies very close to that directly excited by the forcing. The AMM formulation is now a system of 21 equations for the amplitudes of the active modes given by (A.26). The sector mistuning coefficient $m(j)$ now translates directly into a small correction of the blade alone frequency of blade $j$, $\omega_{j} / \omega_{0}=1+m(j) / 2+\ldots$, because the mistuning is implemented as a multiplication of the blade $j$ stiffness matrix by the coefficient $(1+m(j))$.

The maximum amplification of the forced response is computed applying an optimization methorl to the AMM system. The resulting amplification factors 
for $\mathrm{EO}=8$ and $\mathrm{EO}=-8$ are shown in Fig. 7 and Fig. 8, respectively, where the mistuning size is now measured as the maximum of the mistuning distribution relative to the size of the aerodynamic forces,

rescaled mistuning size $=\max (|m(j)|) / c_{a}$

Both curves grow from the tuned value, even for the case $\mathrm{EO}=8$ that has a damping three times smaller than that of $\mathrm{EO}=-8$ (see Fig. 3). This is due to the fact that the mistuning can now couple with more active TWs, generating a stronger increase of the amplitude that overcomes the effect of the higher damping of the other TWs. The maximum amplification obtained is approximately 1.75, smaller than that from expression (13) which gives a value of Amplification $\max _{2}=2.46$. On the other hand, in the case $\mathbf{E O}=-8$ the damping of the forced TW is much higher, and, because of the mistuning induced coupling with $\mathrm{TW}$ with lower damping, the maximum amplification reaches a much larger value of approximately 2.3 , but still smaller than the upper bound (13) Amplification $\max =3.70$.

Each of the square dots in Fig. 7 and Fig. 8 correspond to a calculation of the full FEM of the mistuned blisk for the corresponding optimal mistuned pattern obtained from the optimization of the AMM; the agreement between both results is excellent.

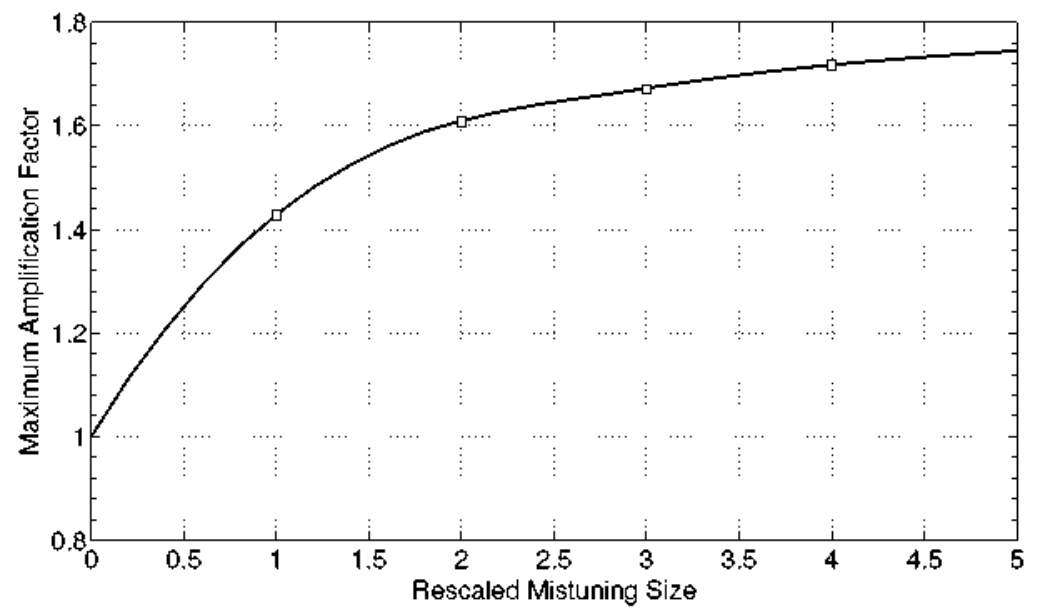

Figure 7: $\mathrm{EO}=8$ forcing. Maximum amplification vs rescaled mistuning size $\max (|m(j)|) / c_{a}$ from the AMM. The squares mark solutions of the full FEM of the mistuned blisk. 


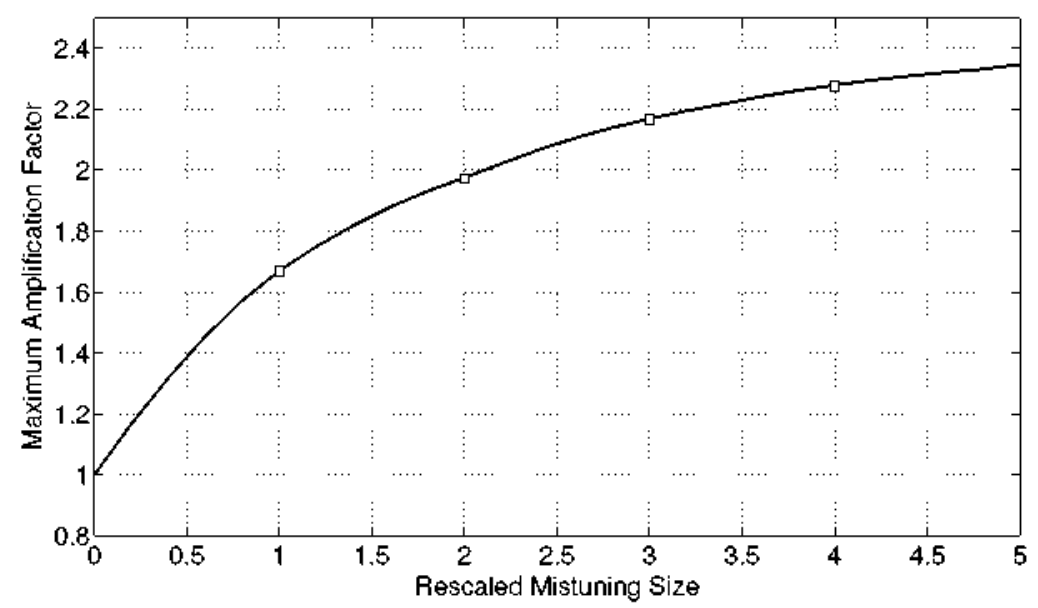

Figure $8: \mathrm{EO}=-8$ forcing. Maximum amplification vs rescaled mistuning size $\max (|m(j)|) / c_{a}$ from the AMM. The squares mark solutions of the full FEM of the mistuned blisk.

The AMM formulation requires the damping and the mistuning to be small. As the damping is increased, the AMM results are expected to deviate from the full FEM results. This effect is illustrated in Fig. 9. The left plot shows the AMM and FEM computed maximum displacement for the $\mathbf{E O}=8$ forcing case and for a rescaled mistuning size 4 (see Fig. 7), and the right plot corresponds to a completely similar situation but with a damping level increased by a factor of $10\left(c_{a}=0.01\right)$. The agreement is excellent for the damping used in this paper, and the results become worse for the increased damping level.

(a)

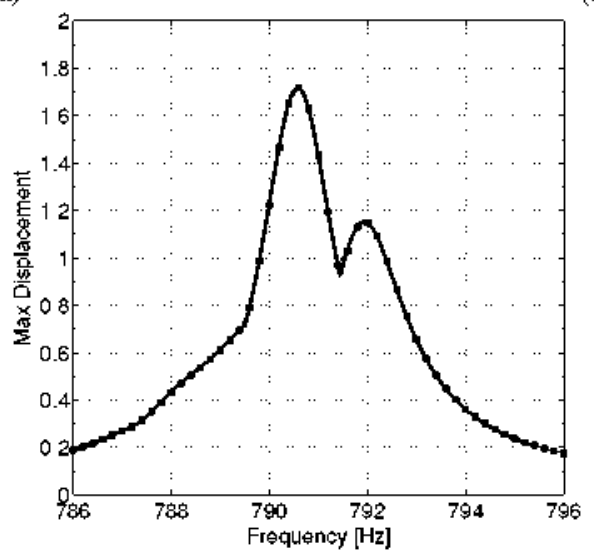

(b)

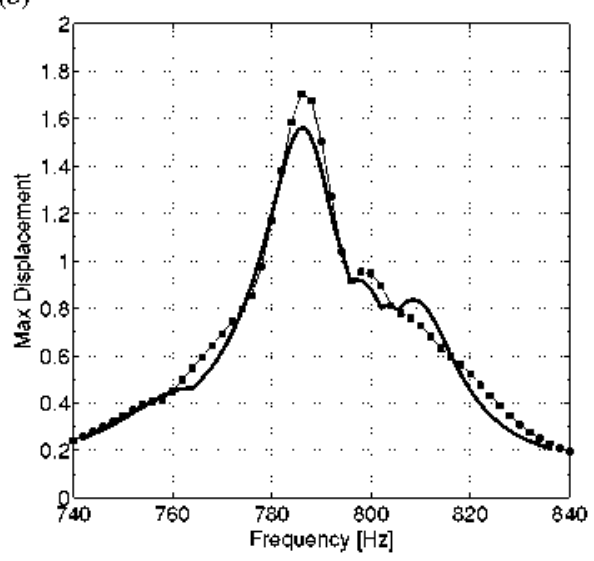

Figure 9: Maximum displacement versus frequency for $\mathrm{EO}=8$ forcing and rescaled mistuning size 4, with $c_{a}=0.001$ (a) and $c_{a}=0.01$ (b). Solid line: FEM results, squares: AMM results. 
It is worth to mention that if there are many active modes present then the system requires higher levels of mistuning to be able to couple all modes and approach the global maximum amplification value (compare Figs. 4 and 6 with Figs. 7 and 8). The AMM properly captures the high sensitivity of the system to the presence of small mistuning imperfections. If the mistuning is further increased then more distant modes (not considered initially as active modes) can be coupled, and higher amplification levels can be probably achieved, but, of course, this regime is not covered by the AMM description whose validity is restricted only to small mistuning values.

On the other hand, the selection of the modes that are considered active is a crucial question for the AMM formulation. The appropriate way to proceed would be to build several versions of the AMM with increasing number of active morles until there are no appreciable changes in the AMM results. This is done and explained in detail in [8]; here the output from the AMM has been just directly compared with the FEM results.

\section{Conclusions}

The AMM formulation has been applied to the analysis of the mistuning effects on the forced response of a stable blisk with damping coming from aerodynamic effects. The AMM is systematically derived from the complete mistuned bladed disk dynamical model using a perturbative procedure based only on the fact that mistuning and damping values are small. The resulting AMM is a very rerluced model that allows to draw the following remarks about how mistuning operates in this configuration.

1. There are two main mechanisms involved that can have opposite effects: (i) the amplification of the response due to the mistuning coupling of the TW modes; typically the more modes involved the higher the response (as in the case of uniform material damping). And (ii) the resulting effective damping is a combination of the damping of the TWs coupled by the mistuning: if the forced TW has small (large) damping then the coupling with the other TWs increases (decreases) the effective damping, and tends to reduce (increase) the vibration amplitude.

2. An upper bound for the maximum amplitude amplification can be computed from the AMM:

$$
\text { Amplification }_{\max }=\frac{1+\sqrt{\xi_{r} \sum_{a} \frac{1}{\xi_{x}}}}{2},
$$

where $\xi_{r}$ is the aerodamping of the TW that is being directly forced, $\xi_{a}$ is the aerodamping of an active TW modes, and the sum goes over all $N_{a}$ active modes. This expression contains the two effects mentioned above in point 1: (i) the number of terms in the sum, and (ii) the ratio of the dampings. 
3. This upper bound is a generalization of Whitehead's expression for the case of TW modes with non-uniform aerodamping. It is very accurate for the forcing of isolated modes, and appears to become more conservative when the number of active modes involved is large (as it also happened with Whitehead's result).

4 . In the case of forcing an isolated TW mode with aerodamping ( $\left.\xi_{+}\right)$smaller than that of its counter-rotating TW $\left(\xi_{-}\right)$, the amplification can be reduced below 1 (that is, below the tuned response), to the value of

$$
\mathbf{A F}=\frac{2 \xi_{+}}{\xi_{+}+\xi_{-}}<1 .
$$

If, on the other hand, the aerodamping of the forced TW is higher ( $\xi_{+}>$ $\xi_{-}$) then the response becomes larger than in the tuned case.

5. In the case of forcing a TW mode that is not isolated and has a large number of nearby modes with similar frequencies there is typically no reduction of the amplification factor. The mistuning couples the TW forced with other active TWs, generating a stronger increase of the amplitude that overcomes the effect of the higher damping of the other TWs.

\section{Acknowledgments}

The authors wish to thank O. Khemiri for providing us with the FEM model of the blisk. This work has been supported by the Spanish Ministerio de Educación y Ciencia under grant DPI-2013-46403-R, and by the Universidad Politécnica de Madrid under grant, GI1514120024.

\section{Appendix}

In this appendix we briefly explain the derivation of the AMM. A more detailed description can be found in [17, 7, 8, 22], where the AMM is successfully applied to the analysis of the effect of mistuning on the flutter characteristics of unstable rotors, and to the computation of the forced response vibration amplitude with frequency and damping mistuning.

The starting point are the general FEM equations for the aeroelastic vibration of a mistuned bladed disk subjected to a pure harmonic forcing with temporal frequency $\omega$

$$
\left(\mathbf{K}+\Delta \mathbf{K}-\omega^{2}(\mathbf{M}+\Delta \mathbf{M})\right) \mathbf{X}=\mathbf{M}_{\text {aero }}(\omega) \mathbf{X}+\mathbf{F},
$$

where the response of the structure is also assumed to be harmonic with frequency $\omega$. The vector $\mathbf{X}$ contains the displacement amplitudes of all independent 
DOFs of the structure, and is arranged by sectors

$$
\mathbf{X}=\left[\begin{array}{c}
X_{1} \\
\vdots \\
X_{j} \\
\vdots \\
X_{N}
\end{array}\right]
$$

with the vector $X_{j}$ containing the displacements of the $m$ DOF associated with sector $j$, and $N$ is the total number of sectors of the bladed disk.

The tuned mass $\mathbf{M}$ and stiffness $\mathbf{K}$ matrices are cyclic matrices given by

$$
\mathbf{M}=\left[\begin{array}{ccccc}
M & M_{c} & 0 & \cdots & M_{c}^{\mathrm{T}} \\
M_{\odot}^{\mathrm{T}} & M & M_{c} & \cdots & 0 \\
& & \ddots & \ddots & \\
M_{c} & 0 & \cdots & M_{c}^{\mathrm{T}} & M
\end{array}\right], \quad \mathbf{K}=\left[\begin{array}{ccccc}
K & K_{\mathrm{c}} & 0 & \cdots & K_{c}^{\mathrm{T}} \\
K_{\odot}^{\mathrm{T}} & K & K_{\odot} & \cdots & 0 \\
& & \ddots & \ddots & \\
K_{c} & 0 & \cdots & K_{c}^{\mathrm{T}} & K
\end{array}\right] \text {, }
$$

where the $m \times m$ sector stiffness and mass matrices $K$ and $M$ are symmetric and the coupling between adjacent sectors is represented by the coupling stiffness and mass matrices $K_{\mathrm{c}}$ and $M_{\mathrm{c}}$, respectively.

The forcing is assumerl to take the form of a pure traveling wave, with frequency $\omega$ and engine order $r$, that is,

$$
\mathbf{F}=\left[\begin{array}{c}
F \mathrm{e}^{\mathrm{i}\left(\frac{2 \pi r}{N}\right) 1} \\
\vdots \\
F \mathrm{e}^{\mathrm{i}\left(\frac{2 \pi r}{N}\right) j} \\
\vdots \\
F \mathrm{e}^{\mathrm{i}\left(\frac{2 \pi r}{N}\right) N}
\end{array}\right]+c \cdot c \cdot
$$

where $F$ contains the in-sector distribution of the forcing, and c.c. stands for the complex conjugate.

The matrix associated with the linearized aerorlynamic forces due to the vibration of the blades, $\mathbf{M}_{\text {atero }}$, depends only on the selected vibration frequency $\omega$. The full computation of $\mathbf{M}_{\text {a ero }}$ would involve: (i) the computation of the stationary flow around the blades, and then (ii) the computation, for each given value of $\omega$, of the solution of the Navier-Stokes equation linearized around the base flow for all linearly independent blade displacements. This task is extremely costly and simply not realizable for any practical realistic bladed disk configuration, but the matrix $\mathbf{M}_{\text {aero }}(\omega)$ is formally kept in the formulation just to progress with the AMM derivation.

Finally, the mistuning is included in the model (A.1) through the mistuning 
stiffness $\Delta \mathbf{K}$ and mass $\Delta \mathbf{M}$ matrices, that take the form

$$
\begin{gathered}
\Delta \mathbf{K}=\left[\begin{array}{cccc}
\Delta K_{1} & 0 & \cdots & 0 \\
0 & \Delta K_{2} & \cdots & 0 \\
& & \ddots & \\
0 & 0 & 0 & \Delta K_{N}
\end{array}\right], \Delta \mathbf{M}=\left[\begin{array}{cccc}
\Delta M_{1} & 0 & \cdots & 0 \\
0 & \Delta M_{2} & \cdots & 0 \\
& & \ddots & \\
0 & 0 & 0 & \Delta M_{N}
\end{array}\right],(A, \\
\text { wit } \Delta \mathrm{h} \quad \sum_{j=1}^{N} \Delta K_{j}=0, \sum_{j=1}^{N} \Delta M_{j}=0 \\
\text { and } \Delta K_{j}=\Delta K_{j}^{\mathrm{T}}, \Delta M_{j}=\Delta M_{j}^{\mathrm{T}} \text { for } j=1 \ldots N .
\end{gathered}
$$

Note that we are not considering mistuning in the coupling between sectors, and that, in order to avoid any ambiguities, we have set the sector to sector mistuning average to zero (see eq. (A.6)), which is equivalent to define the tuned sector as the average of the mistuned sectors.

The system (A.1) is transformed from the displacements description, $\mathbf{X}$, to the tuned TW amplitudes, $\mathbf{A}$, using the change of variables:

$$
\mathbf{X}=\mathbf{P A}, \quad \mathbf{A}=\left[\begin{array}{c}
A_{1} \\
\vdots \\
A_{k} \\
\vdots \\
A_{N}
\end{array}\right]
$$

where

$$
\mathbf{P}=\frac{1}{\sqrt{N}}\left[\begin{array}{ccc}
P_{1} \mathrm{e}^{\mathrm{i}\left(\frac{2 \pi 1}{N}\right) 1} & \ldots & P_{N} \mathrm{e}^{\mathrm{i}\left(\frac{2 \pi N}{N}\right) 1} \\
\vdots & & \vdots \\
P_{1} \mathrm{e}^{\mathrm{i}\left(\frac{2 \pi 1}{N}\right) j} & \ldots & \left.P_{N} \mathrm{e}^{\mathrm{i}\left(\frac{2 \pi N}{N}\right) j}\right) \\
\vdots & & \vdots \\
P_{1} \mathrm{e}^{\mathrm{i}\left(\frac{2 \pi 1}{N}\right) N} & \ldots & P_{N} \mathrm{e}^{\mathrm{i}\left(\frac{2 \pi N}{N}\right) N}
\end{array}\right]
$$

and the block matrices $P_{k}$ of the matrix $\mathbf{P}$ contain all tuned elastic eigenmodes $Z_{k p}$ corresponding to nodal diameter equal to $k$ :

$$
P_{k}=\left[Z_{k 1}\left|Z_{k 2}\right| \cdots\left|Z_{k p}\right| \cdots \mid Z_{k m}\right] .
$$

The system (A.1) in the TW basis takes the following form:

$$
\begin{gathered}
\left(\left[\begin{array}{ccc}
\Omega_{1}^{2}-\omega^{2} I & \ldots & 0 \\
\vdots & \ddots & \vdots \\
0 & \cdots & \Omega_{N}^{2}-\omega^{2} I
\end{array}\right]+\Delta\right)\left[\begin{array}{c}
A_{1} \\
\vdots \\
A_{N}
\end{array}\right]= \\
=\mathbf{P}^{\mathrm{H}} \mathbf{M}_{\text {aero }}(\omega) \mathbf{P}\left[\begin{array}{c}
A_{1} \\
\vdots \\
A_{N}
\end{array}\right]+\mathbf{P}^{\mathrm{H}} \mathbf{F}
\end{gathered}
$$


where the identity matrix $I$ and the $\Omega_{k}^{2}$ matrices come from the transformed tuned mass and stiffness matrices, respectively. The $\Omega \frac{2}{k}$ matrices just have the squares of the eigenfrequecies $\omega_{k p}$ for nodal diameter $k$ on the diagonal:

$$
\Omega_{k}^{2}=\left[\begin{array}{cccc}
\omega_{k 1}^{2} & 0 & \cdots & 0 \\
0 & \omega_{k 2}^{2} & \ddots & \vdots \\
\vdots & \ddots & \ddots & 0 \\
0 & \cdots & 0 & \omega_{k m}^{2}
\end{array}\right]
$$

Note that the contribution of the tuned mass and stiffness matrices becomes diagonal in the $\mathrm{TW}$ basis.

The forcing $\mathbf{F}$ has $\mathbf{T W}$ form with nodal diameter $k=r$ (see eq. (A.4)), and, consequently, the left multiplication by the matrix $\mathbf{P}^{\mathrm{H}}$ produces a block vector with null entries except for the block corresponding to the engine order $r$ (recall that the TWs with different number of nodal diameters are orthogonal):

$$
\mathbf{P}^{\mathrm{H}} \cdot \mathbf{F}=\left[\begin{array}{c}
0 \\
\vdots \\
P_{r}^{\mathrm{H}} \cdot F \\
\vdots \\
0
\end{array}\right]
$$

The linearized aerorlynamic flow on the tuned rotor has also cyclic symmetry because it is identical on all sectors. This leads, again formally, to a block diagonalization of the aerodynamic matrix $\mathbf{M}_{\text {aero }}$ in the TW basis:

$$
\mathbf{P}^{\mathrm{H}} \mathbf{M}_{\text {aero }}(\omega) \mathbf{P}=\left[\begin{array}{cccc}
M_{\text {aero } 1}(\omega) & 0 & \cdots & 0 \\
0 & M_{\text {aero2 }}(\omega) & \ddots & \vdots \\
\vdots & \ddots & \ddots & 0 \\
0 & \cdots & 0 & M_{\text {aero } N}(\omega)
\end{array}\right]
$$

The mistuning of the aerodynamic forces is neglected in this analysis because it is typically of higher order; it is a small correction of the already small aerodynamic effects. Stronger airfoil morlifications would be required to make aerodynamic mistuning relevant (as in the case of leading edge blending [23]), but this is not the situation considered here.

And finally, the mistuning correction matrix $\Delta$ that contains all the mistuning terms is defined as:

$$
\boldsymbol{\Delta}=\mathbf{P}^{\mathrm{H}}\left(\Delta \mathbf{K}-\omega^{2} \Delta \mathbf{M}\right) \mathbf{P}
$$

To further understand the structure of this matrix it is convenient to expand in discrete Fourier series the mistuning distribution along the $N$ sectors of the 
bladed disk (see (A.5))

$$
\begin{aligned}
& \Delta K_{j}=\sum_{k=1}^{N} \Delta K_{k}^{\mathrm{F}} \mathrm{e}^{\mathrm{i}\left(\frac{2 \pi k}{N}\right) j} \text { and } \\
& \Delta M_{j}=\sum_{k=1}^{N} \Delta M_{k}^{\mathrm{F}} \mathrm{e}^{\mathrm{i}\left(\frac{2 \pi k}{N}\right) j} \text { for } j=1 \ldots N
\end{aligned}
$$

The $k$ Fourier coefficients, $\Delta K_{k}^{\mathrm{F}}$ and $\Delta M_{k}^{\mathrm{F}}$, indicate the amount of harmonic wave with $k$ nodal diameters in the mistuning pattern, and verify the following conditions (which are just direct consequences of (A.6) and (A.7))

$$
\begin{gathered}
\Delta K_{N}^{\mathrm{F}}=0, \Delta M_{N}^{\mathrm{F}}=0 \text { and } \Delta K_{k}^{\mathrm{F}}=\overline{\Delta K_{-k}^{\mathrm{F}}}, \quad \Delta M_{k}^{\mathrm{F}}=\overline{\Delta M_{-k}^{\mathrm{F}}}, \\
\Delta K_{k}^{\mathrm{F}}=\left(\Delta K_{k}^{\mathrm{F}}\right)^{\mathrm{T}}, \Delta M_{k}^{\mathrm{F}}=\left(\Delta M_{k}^{\mathrm{F}}\right)^{\mathrm{T}}, \quad \text { for } \quad k=1 \ldots N
\end{gathered}
$$

Note that the Fourier mode $-k$ is the same as the mode $N-k$ since the arldition of $N$ leaves all expressions unchanged. After inserting the above expressions (A.16) and (A.17) into the mistuning correction matrix $\Delta$, it takes the form

$$
\boldsymbol{\Delta}=\left[\begin{array}{cccc}
0 & \Delta_{12} & \ldots & \Delta_{1 N} \\
\Delta_{21} & 0 & \ldots & \Delta_{2 N} \\
\vdots & \vdots & \ddots & \vdots \\
\Delta_{N 1} & \Delta_{N 2} & \ldots & 0
\end{array}\right]
$$

where each block is related to the Fourier components of the block mistuning matrices $\Delta K_{j}$ and $\Delta M_{j}$ by:

$$
\Delta_{k k^{\prime}}=P_{k}^{\mathrm{H}}\left(\Delta K_{k-k^{\prime}}^{\mathrm{F}}-\omega^{2} \Delta M_{k-k^{\prime}}^{\mathrm{F}}\right) P_{k^{\prime}}
$$

The idea of the AMM methodology is to take into account the fact that the mistuning, the forcing and the aerodynamic effects are just small corrections of the elastic tuned dynamics, and apply an asymptotic perturbative procedure to obtain a rerluced model of the system (see $[17,7,8]$ ). Near a resonance, the only TW modes that have to be retainerl are those with frequency close to the tuned resonant frequency (active modes). The rest of the modes (passive morles), with frequencies that are well apart from the resonant frequency, have very small amplitudes and can be neglecterl in a first order approximation.

The AMM is basically the reduced model that results from keeping only the active modes in system (A.11), which obey equations of the form

$$
\left(\omega_{a}^{2}-\omega^{2}\right) A_{a}+\sum_{a a^{\prime} \neq a} \delta_{a a^{\prime}} A_{a^{\prime}}-\left[M_{\mathrm{aerok}}(\omega)\right]_{a a} A_{a}=F_{a},
$$

where $A_{a}$ is the amplitude of the active mode considered, and $A_{a^{\prime}}$ stands for the amplitudes of the rest of the active modes. The different terms in the above erfuation can be further simplified: (i) close to the tunerl resonant frequency $\omega_{r}$, 
both the modal frequency and the forcing frequency can be written as $\omega_{a}=$ $\omega_{r}\left(1+\Delta \omega_{a}\right)$ and $\omega=\omega_{r}(1+\Delta \omega)$, and the first coefficient can be expanded as

$$
\left(\omega_{a}^{2}-\omega^{2}\right) \cong 2 \omega_{r}^{2}\left(\Delta \omega_{a}-\Delta \omega+\ldots\right)
$$

(ii) the mistuning coupling coefficients $\delta_{a a^{\prime}}$ can be also approximated as

$$
\begin{aligned}
\delta_{a a^{\prime}} & =Z_{a}^{\mathrm{H}}\left(\Delta K_{a-a^{\prime}}^{F}-\omega^{2} \Delta M_{a-a^{\prime}}^{F}\right) Z_{a^{\prime}} \\
& \cong Z_{a}^{\mathrm{H}}\left(\Delta K_{a-a^{\prime}}^{F}-\omega_{r}^{2} \Delta M_{a-a^{\prime}}^{F}\right) Z_{a^{\prime}},
\end{aligned}
$$

(iii) the aerodynamic coefficient $\left[M_{\text {aerok }}(\omega)\right]_{a a}$ can be also expressed in terms of the frequency correction, $\eta_{a}$, and the aerodynamic damping, $\xi_{a}$,

$$
\left[M_{\text {aerok }}(\omega)\right]_{a a}=-2 \omega_{a}^{2}\left(\eta_{a}+\mathrm{i} \xi_{a}\right)
$$

and (iv) the forcing term $F_{a}$ is zero everywhere except at the active mode that is being directly forced, $r$, where it takes the value

$$
F_{r}=2 \mathrm{i} \omega_{r}^{2} \xi_{r}
$$

which has been set to fix the value of the maximum tuned response amplitude to 1 .

The AMM assumes that, in first approximation, only the active modes contribute to the mistuned response of the system, and it is constructed just by collecting the equations (A.21) for all $N_{a}$ active modes

$$
\left[\begin{array}{cccc}
d_{a} & & & \\
& \ddots & & \\
D^{\mathrm{H}} & & \ddots & \\
& & & d_{a^{\prime}}
\end{array}\right]\left[\begin{array}{c}
A_{a} \\
\vdots \\
\vdots \\
A_{a^{\prime}}
\end{array}\right]=\left[\begin{array}{c}
0 \\
2 \mathrm{i}_{r}^{2} \xi_{r} \\
\vdots \\
0
\end{array}\right]
$$

where $A_{a}, \ldots, A_{a}$ are the amplitudes of the active modes. The diagonal terms on the left hand side are given by

$$
d_{a}=2 \omega_{r}^{2}\left(\Delta \omega_{a}-\Delta \omega\right)+2 \omega_{a}^{2}\left(\eta_{a}+\mathrm{i} \xi_{a}\right)
$$

and the off-diagonal mistuning elements in $D$ are defined by the following expression:

$$
D_{a a^{\prime}}=Z_{a}^{\mathrm{H}}\left(\Delta K_{a-a^{\prime}}^{\mathrm{F}}-\omega_{r}^{2} \Delta M_{a-a^{\prime}}^{\mathrm{F}}\right) Z_{a^{\prime}}
$$

which indicates that the effect of mistuning is just to couple TWs with different nodal diameters: the TW with $k$ nodal diameters is coupled to the TW with $k^{\prime}$ nodal diameters precisely through the $\left(k-k^{\prime}\right)$ Fourier mode of the mistuning distribution. 


\section{References}

[1] M. Castanier, C. Pierre, Modeling and analysis of mistuned bladed disk status and emerging directions, Journal of Propulsion and Power 22 (2) (2006) $384-396$.

[2] D. Hemberger, D. Filsinger, H. Baner, Investigations on maximum amplitude amplification factor of real mistuned bladed structures, Proceedings of the ASME Turbo Expo 2012 GT2012-68084.

[3] B. Cowles, High cycle fatigue in aircraft gas turbines-an industry perspective, International Journal of Fracture 80 (1996) 147-163.

[4] T. Nicholas, Critical issues in high cycle fatigue, International Journal of Fatigue 21 (1999) S221-S231.

[5] D. Thomsom, D. Griffin, The national turbine engine high cycle fatigue program, Global Gas Turbine News 39 (1999) 14-17.

[6] D. Whitehead, Effect of mistuning on the vibration of turbomachine blades induced by wakes, Journal of Mechanical Engineering Science 8 (1) (1966) $15-21$.

[7] C. Martel, R. Corral, Asymptotic description of maximum mistuning anplification of bladed disk forced response, Journal of Engineering for Gas Turbines and Power 131 (2009) 022506.

[8] O. Khemiri, C. Martel, R. Corral, Forced response of mistuned bladed disks: Asymptotic description and fem validation, AIAA Journal of Propulsion and Power 30 (2013) 397-406.

[9] Y. Kaneko, K. Mori, H. Ooyama, Resonant response of mistuned bladed disk expressed by vibratory stress, Proceedings of the ASME Turbo Expo 2016 GT2016-56053.

[10] J. Kielb, R. Abhari, Experimental study of aerodynamic and structural damping in a full-scale rotating turbine, Journal of Engineering for Gas Turbines and Power 125 (2003) 102-112.

[11] E. Petrov, A method for forced response analysis of mistuned bladed disks with aerodynamic effects included, Journal of Turbomaxhinery 132 (2010) 062502-1-10.

[12] E. Petrov, Reduction of forced response levels for bladed disks by mistuning: Overview of the phenomenon, Journal of Turbomaxhinery 133 (2011) 072501-1-10.

[13] H. Schoenenborn, M. Junge, U. Retze, Contribution to free and forced vibration analysis of an intentionally mistuned blisk, Proceedings of the ASME Turbo Expo 2012 GT2012-68683. 
[14] B. Berirow, T. Giersh, A. Kühhorn, J. Nipkau, Forced response analysis of a mistuned compressor blisk, Journal of Engineering for Gat Turbines and Power 136 (2014) 062507-1-13.

[15] B. Beirow, A. Kühhorn, F. Figaschewsky, J. Nipkau, Effect of mistuning and damping on the forced response of a compressor blisk rotor, Proceedings of the ASME Turbo Expo 2015 GT2015-42036.

[16] F. Bessem, R. Kielb, N. Key, Forced response sensitivity of a mistuned rotor from an embedded compressor stage, Proceedings of the ASME Turbo Expo 2015 GT2015-43032.

[17] C. Martel, R. Corral, J. Llorens, Stability increase of aerodynamically unstable rotors using intentional mistuning, Journal of Turbomachinery 130 (2008) 011005.

[18] Y. Chan, D. Ewins, The amplification of vibration response leves of mistuned bladed disks: its consequences and its distribution in specific situations, Journal of Engineering for Gas Turbines and Power 133 (2011) $102502-1-8$.

[19] R. Bladh, M. Castanier, C. Pierre, Component-mode-based reduced order modeling techniques for mistuned bladed disks-part i: Theoretical models, Journal of Engineering for Gas Turbines and Power 123 (2001) 89-99.

[20] R. Bladh, M. Castanier, C. Pierre, Component-mode-based reduced order modeling techniques for mistuned bladed disks-part ii: Application, Journal of Engineering for Gas Turbines and Power 123 (2001) 100-108.

[21] D. Ewins, The effects of detuning upon the forced vibrations of bladeddisks, Journal of Sound and Vibration 9 (1) (1969) 65-79.

[22] O. Khemiri, C. Martel, R. Corral, Asymptotic description of damping mistuning effects on the forced response of turbomachinery bladed disks, Journal of Sound and Vibration 332 (2013) 4998-5013.

[23] K. Ekici, R. Kielb, K. Hall, The effect of aerodynamic asymmetries on turbomachinery flutter, Journal of Fluids and Structures 36 (2013) 1-17. 